\title{
Die Migrantin retten!?
}

\section{Zum vertrackten Verhältnis von Geschlechtergewalt, Rassismus und Handlungsmacht}

\author{
María do Mar Castro Varela · Nikita Dhawan
}

(C) Der/die Autor(en) 2016. Dieser Artikel ist eine Open-Access-Publikation.

Zusammenfassung Der vorliegende Beitrag beschäftigt sich mit den zwingenden feministischen Fragen nach Geschlechtergewalt, Verletzlichkeit, Handlungsmacht, deren Verknüpfungen und den Dilemmas, die jene spüren, die sexistische Gewalt, insbesondere innerhalb rassifizierter Communities, zu adressieren suchen. Dabei wird eine postkolonial-feministische Perspektive eingenommen, die eine Sicht auf Migration und Flucht erlaubt, bei der gleichzeitig feministische Mainstream-Annahmen mit Blick auf die andere Frau einer Kritik unterzogen werden. Angelehnt an Judith Butler wird dabei argumentiert, dass neue Formen kollektiver Handlungsmacht paradoxerweise gerade dort entstehen, wo eine spezifische Vulnerabilität verortet ist. Wenn auch eine sichtbare Verletzlichkeit häufig zu paternalistischen (Schutz-)Maßnahmen der hegemonialen Mehrheit führt, die gewaltvolle Geschlechterdynamiken stabilisieren, so können im Raum der Verwundbarkeit, wie Judith Butler feststellt, auch neue Formen von Handlungsmacht entstehen. Es ist in der Tat möglich, Opfer und zeitgleich Handlungsmächtige zu sein.

Schlüsselwörter Geschlechtergewalt · Feminismus/Rassismus · Migration/Flucht · Dekolonisierung

\footnotetext{
M. Castro Varela

Alice Salomon Hochschule, Alice-Salomon-Platz 5, 12627 Berlin, Deutschland

E-Mail: castrovarela@posteo.de

N. Dhawan $(\bowtie)$

Institut für Polikwissenschaft, Universität Innsbruck, Universitätsstraße 15, 6020 Innsbruck,

Österreich

E-Mail: Nikita.Dhawan@uibk.ac.at
} 


\title{
Rescuing the female migrant!?
}

On the tricky relation between gendered violence, racism and agency

\begin{abstract}
The contribution engages with the pivotal feminist issues of gendered violence, vulnerability, and agency and the concomitant dilemmas that emerge when addressing the problem of gendered and sexualized violence particularly in migrant and refugee communities. Drawing on a postcolonial-feminist perspective, the effort is to critically outline the mainstream construction of the other woman in discussions on migration and flight. As argued by Judith Butler, while new modes of collective agency can emerge by drawing on vulnerability as a site of political agency, it can also justify paternalistic protection reinforcing coercive processes of gender formation. It is indeed possible to simultaneously be vulnerable and have agential power.
\end{abstract}

Keywords Gendered violence $\cdot$ Racism/sexism $\cdot$ Migration/refuge $\cdot$ Decolonization

Der vorliegende Beitrag beschäftigt sich mit den zwingenden feministischen Fragen nach den Verknüpfungen von Geschlechtergewalt, Verletzlichkeit und Handlungsmacht und den Dilemmas, die jene spüren, die sexistische Gewalt innerhalb rassifizierter Communities adressieren müssen. Dabei wird eine postkolonial-feministische Perspektive eingenommen, die eine Sicht auf Migration und Flucht erlaubt, die Annahmen des feministischen Mainstreams gegenüber der anderen Frau kritisch hinterfragt. Angelehnt an Judith Butler (2004) argumentieren wir, dass kollektive Handlungsmacht paradoxerweise gerade dort entstehen kann, wo eine spezifische Verletzlichkeit verortet ist. Selbst wenn sichtbare Verletzlichkeiten nicht selten paternalistische (Schutz-)Maßnahmen zur Folge haben, die zudem gewaltvolle Geschlechterdynamiken stabilisieren, so können im Raum der Verwundbarkeit auch neue Formen von Handlungsmacht entstehen. Kurzum: Es ist möglich, Opfer und zeitgleich Handlungsmächtige zu sein. Und dies ist nur scheinbar paradox. So können wir im Zusammenhang mit der hier interessierenden Subjektposition „hilfsbedürftige Migrantin“ beobachten, dass jede Thematisierung von Geschlechtergewalt das stereotype Bild patriarchaler, gewalttätiger migrantischer Männer und Communities $^{1}$ stabilisiert und in der Folge rassistische Praxen - manches Mal nolens volens - mobilisiert. Eine vertrackte Situation, denn die Nicht-Thematisierung von Geschlechtergewalt kann in Anbetracht der realen Gewalt kaum als sinnvoll angesehen werden. Um die sozialen Dynamiken besser verstehen und einordnen zu können, werfen wir einen kurzen Blick in die Geschichte der Intersektion von sexistischer und rassistischer Gewalt. Schließlich wird dadurch auch die strukturelle Beziehung zwischen denen bestimmt, die über eine unhinterfragte Zugehörigkeit verfügen, und

\footnotetext{
1 Wie vehement sich das unreflektierte Vorurteil der ,migrantischen Machos' (vgl. auch Gemende u. a. 2007) hält, kann an den aktuellen Debatten zu aus Syrien Geflüchteter nachgezeichnet werden. Immer wieder werden etwa junge männliche Syrer repräsentiert, als seien ihre Handlungen weniger rational und stattdessen mehr vom überschäumenden Testosteron bestimmt.
} 
jenen, die immer wieder zu Anderen gemacht werden: Migrant_innen, Geflüchtete, diasporische Subjekte, ehemalig Kolonisierte, Muslim_innen etc.

Dass die Geschlechterbeziehungen der Anderen Westeuropas seit der Kolonialzeit als das Symbol für die Rückständigkeit und den Barbarismus der (ehemals) Kolonisierten galten, ist kaum mehr zu bestreiten. Die Viktimisierung der anderen Frau hat im Westen aber nicht nur eine lange Tradition, sie erfüllt auch wichtige Funktionen (vgl. hierzu Mohanty 1988; Spivak 1994). Etwas simplifizierend lässt sich dies als ein Prozess beschreiben, der wie folgt aussieht: Die Festschreibung der anderen Frau als Opfer ermöglicht es einerseits der hegemonialen Frau, sich als emanzipiert und modern zu beschreiben. Anderseits werden parallel zur unkritischen Viktimisierung der anderen Frau Interventionen legitimiert, die gegen jene humanistischen Werte verstoßen, die Europa angeblich erst zu Europa machen. Der scheinbar emanzipatorische Diskurs eröffnet einen anti-aufklärerischen Raum, in dem Aufklärung schlicht das ist, was Europa hat, während die Anderen dieser immer nur hinterherhinken können. Machtanalytische Perspektiven können diese Verquickung immerhin soweit entwirren, dass dies als Verbindung lesbar wird ${ }^{2}$. Dann lässt sich etwa fragen: Warum hält sich das westliche Vorurteil von der unterdrückten muslimischen oder geflüchteten Frau mit solcher Vehemenz? Welche Rolle spielen postkoloniale Positionen im feministischen Diskurs des Westens? Wie ist die Beziehung zwischen Rassismus und Sexismus konkret zu beschreiben $?^{3}$ Das sind einige der Fragen, die den Beitrag rahmen sollen. Die Beantwortung kann hier nur fragmentiert gelingen, doch geht es auch eher darum, auf ein wirkmächtiges Dilemma aufmerksam zu machen, als darum, einfache Lösungen zu propagieren - die immer zwangsläufig problematisch sind. Postkoloniale Gewaltverhältnisse sind komplex. Sie verweigern sich simplen Lösungen und nötigen uns dazu, Ambivalenzen auszuhalten.

\section{Geschlechtergewalt und Verletzlichkeit}

Die feministische Kritik weist eine lange Geschichte diskursiver Interventionen auf einen eng ausgelegten Gewaltbegriff auf. Eine enge Auslegung, die nur körperliche Verletztung als Gewalt anerkennt, wird zurückgewiesen und herausgefordert. Die Debatten um Gewalt in der Familie ermöglichten beispielsweise, den bis dahin geradezu unantastbaren Raum der Familie zu analysieren und die Gewalt, die in diesem von der Öffentlichkeit oft unbemerkt stattfindet, zu skandalisieren: etwa

\footnotetext{
${ }^{2}$ Eine der Konsequenzen dieser Herrschaftstrategie ist eine spezifische Konsumtionspolitik, die das Selbstbewusstsein der Europäer_innen stärkt und die Anderen in einem ständigen Verteidigungsmodus zurücklässt.

3 Wie ist etwa zu erklären, dass EMMA, die wohl bekannteste deutsch-feministische Frauenzeitschrift, in aktuellen Beiträgen, die sich mit dem Thema Flucht beschäftigen, von „frauenverachtenden Traditionen“ schreiben kann und zudem noch bemerkt, durch die Fluchtbeweungen seien Menschen- und Frauenrechte in Gefahr. Ein ähnlicher Diskurs wird auch vom Österreichischen Bundesministerium für Inneres mobilisiert, wenn dieses in einem „Refugee-Guide“ die Grundregeln des Zusammenlebens in Österreich erläutert und dabei betont, dass in Österreich Frauen und Männer die gleichen Rechte hätten und die Geflüchtete diese Werte lernen und akzeptieren müssten (vgl. Republik Österreich, Bundesministerium für Inneres 2016).
} 
sexuelle Gewalt oder Vergewaltigung in der Ehe. Themen, die lange Zeit als Tabu erachtet wurden. Hervorgegangen sind daraus rechtliche Forderungen wie die nach der Anerkennung unterschiedlicher Formen von vergeschlechtlichen Gewalttaten als Straftatbestand, aber auch Selbsthilfeorganisationen im Bereich sexueller Gewalt entstanden in Konsequenz dieser Debatten. Breit rezipiert wurde in den feministischen Diskussionen Johan Galtungs Konzept der „strukturellen Gewalt“ (1988) wie auch das Foucault'sche der ,epistemischen Gewalt“, welches insbesondere innerhalb der feministisch-postkolonialen Theorie eine breite Rezeption erfuhr. Ersteres beschreibt gewaltförmige soziale Strukturen - beispielsweise Bildungssysteme, die nach wie vor insbesondere proletarische Migrant_innen diskriminieren und ausgrenzen (vgl. Castro Varela 2015). Die epistemische Gewalt ermöglicht supplementierend eine Analyse der Wissensproduktionen und fokussiert die Beziehung zwischen Wissen und Macht wie auch ein differenziertes Verständnis von Subjektproduktion dadurch möglich wird. Relevante Fragen sind etwa: Welches Wissen findet Anerkennung? Wer profitiert von welchem Wissen? Und wie wird nicht-hegemoniales Wissen disqualifiziert?

Alle diese Diskussionen und begrifflichen Erweiterungen ermöglichen es, Gewalt differenziert zu artikulieren und sie damit politisch adäquater zu adressieren. Die feministische Neukalibrierung des Gewaltkonzepts ließ es darüber hinaus zu, selbst die Nicht-Intelligibilität von Gewalt als Gewalt zu erfassen. So lässt sich fragen, warum es nicht möglich ist, spezifische Gewaltphänomene öffentlich zu artikulieren. Wie kommt es zur De-Thematisierung von Gewalt? Konkret ließe sich beispielsweise fragen, warum Gewalt, die innerhalb der Institution Ehe beziehungsweise in den Familien stattfindet, nicht als solche benannt werden darf. Zweifelsohne profitieren die Täter_innen, während die Opfer erneut viktimisiert werden, indem ihnen die Möglichkeit genommen wird, die Taten öffentlich zu denunzieren. Damit verknüpft ist eine normative Gewalt, die Butler (1999, S. xx) zufolge von den Normen der Gesellschaft selber ausgeht und festlegt, was als nicht-normativ markiert wird.

Eine besondere Herausforderung innerhalb der feministischen Auseinandersetzungen stellt darüber hinaus nach wie vor die Biologisierung von Gewalt dar, die sich in Argumentationstaktiken nachweisen lässt, die von einer quasi natürlichen Verletzlichkeit von Frauen ausgehen und die potentielle Gewalttätigkeit von Männern mit deren biologischer Disposition erklärt. Frühe feministische Theorien beharrten noch auf einer strikten Geschlechterdifferenz und vertraten die Ansicht, dass Frauen jenseits aller Unterschiede eine Gewaltgeschichte teilen (siehe etwa Daly 1978). ${ }^{4}$ Während das Patriarchat als ein weltweites und prinzipiell gleich wirkendes männliches Gewaltsystem gegen Frauen beschrieben wurde, wurden Frauen als

\footnotetext{
4 Anhand der Rezeptionsgeschichte von „Gyn/Ecology“ der US-amerikanischen feministischen Theologin Mary Daly (1978) lässt sich die Problematik solcherlei Vorstellungen gut verdeutlichen. Die radikalfeministischen Ausführungen Dalys wurden seinerzeit von der schwarzen Feministin Audre Lorde zurückgewiesen. Unter anderem weil die Geschichte und Erfahrungen Schwarzer Frauen darin ausgelöscht werden. So schreibt Lorde in einem offenen Brief ,What you excluded from Gyn/Ecology dismissed my heritage and the heritage of all other noneuropean women, and denied the real connections that exist between all of us“ (Lorde [1979] (1984)). Des Weiteren wurde das Buch als transphobisch bezeichnet, weil Daly Geschlechtsanpassungen als „Frankenstein Phänomen“ beschreibt. Viele Feministinnen allerdings feierten die universale Darstellung von der männlichen Gewalt gegen Frauen.
} 
monolithische Einheit gesehen, die im Patriarchat Gewalt erleidet. ${ }^{5}$ Im deutschsprachigen Raum war es insbesondere die Frauenforschung zum Nationalsozialismus und Kolonialismus, die in den 1980er-Jahren den entscheidenden Impuls in Richtung Veränderung der Auseinandersetzungen mit „Geschlecht und Gewalt“ setzte (etwa Mamozai 1982). Die These von der Mittäterschaft von Frauen besagt dabei, dass Frauen Gewaltverhältnissen nicht wie einer von außen kommenden Macht gegenüberstehen, sondern dass sie auch an ihnen mitwirken, von diesen profitieren (vgl. Thürmer-Rohr 1989). Wenn auch ein Sprechen von Mittäterschaft immer noch einen Unterschied zwischen Tätern und Mittäterinnen konstatierte, so wurde zumindest anerkannt, dass Frauen nicht nur Opfer gesellschaftlicher Verhältnisse sind. Frauen, so ist spätestens seit diesen Interventionen klar, sind verletzungsoffen und zu Gewalt fähig. Je nach sozialer Positionierung können sie auch Privilegien aus machtvollen Gewaltverhältnissen ziehen. In den Kolonien etwa konnte die europäische Frau, auch wenn sie selbst keinen Zugang zu Bildung und/oder ökonomischen Ressourcen hatte, über Sklav_innen verfügen und ihre Respektabilität aus ihrer hegemonial zuerkannten weißen Vorherrschaft ziehen. In ähnlicher Weise gelingt es den dominanten Westeuropäerinnen heute, sich über muslimische Migrantinnen zu erheben, indem sie diesen Emanzipationsfähigkeit absprechen. Muslimische Weiblichkeit und Freiheit scheinen im hegemonialen westlich-feministischen Denken inkommensurabel zu sein. Auch aufgrund dieser Diskurse scheint ein Sprechen von einem „imperialistischen Feminismus“ (Spivak 1994) durchaus gerechtfertigt. Die Mittäterschaftsthese führte zudem zur Sichtbarmachung des nie wirklich vorhandenen Konsenses innerhalb der feministischen Theorie und Praxis (vgl. ebd.). ${ }^{6}$ Mit der Thematisierung von Rassismus und Antisemitismus im weitesten Sinne konnte nicht mehr an der These festgehalten werden, die Geschlechterdifferenz sei das entscheidende Element zwischenmenschlicher Gewalt. ${ }^{7}$

Aufbauend auf diesen Überlegungen wird im Nachfolgenden Geschlechtergewalt in fluchtmigrantischen ${ }^{8}$ Communities skizziert, ihre gleichzeitige Thematisierung und De-Thematisierung problematisiert und die Notwendigkeit einer intersektionalen Betrachtungsweise von Gewalt formuliert.

\footnotetext{
5 Eine Auseinandersetzung mit Heteronormativiät und einer Zweigeschlechterordnung ist hier kaum nachweisbar. Erst mit einer stärkeren Rezeption poststrukturalistischer Ansätze in feministischen Schriften und der schrittweisen Etablierung der Queer Studies werden die Kategorien von „Mann“ und „Frau“ produktiv hinterfragt.

${ }^{6}$ Hier sollte angemerkt werden, dass das Sprechen von einer universalen Frauenbewegung immer schon Angriffen ausgesetzt war. Die erste deutsche Frauenbewegung etwa spaltete sich in eine bürgerliche, radikale, jüdische und proletarische Frauenbewegung, die nicht nur unterschiedliche Flügel der Bewegung vertraten, sondern deren Politiken und Ziele durchaus antagonistisch waren. Es ist mühsam, den Beginn der kritischen Differenzdebatten zu markieren, aber ganz sicher liegt dieser weit vor der Einführung des Intersektionalitätsansatzes in den 1990er-Jahren.

7 Die Debatten im deutschsprachigen Raum knüpfen dabei kaum zufällig an die US-amerikanischen Interventionen afroamerikanischer Feministinnen an, die in den 1980er-Jahren den Rassismus der weißen Frauenbewegung offen legten und einen Black Feminism inaugurierten, der weitreichende politische und theoretische Debatten zur Folge hatte (etwa Lorde 1984; Hill Collins 1990).

8 Die Bezeichnung ,fluchtmigrantisch“ versucht die Verschlungenheit von Flucht und Migration zu betonen und dabei gleichzeitig die Differenz zwischen den beiden Erfahrungen nicht zu löschen.
} 


\section{Geschlecht und (De-)Kolonisierung}

In Zeiten eines zugespitzten globalen antimuslimischen Klimas müssen wir uns fragen, ob über Gewalt innerhalb muslimischer Communities überhaupt öffentlich gesprochen werden kann, ohne dass dieses Sprechen gewalttätigen Hass schürt. Doch kann andersherum ein Verschweigen sinnvoll sein, wenn dabei doch die faktische Geschlechtergewalt relativiert wird? Zurzeit wird beispielsweise jede Nachricht über Gewalt in den Unterbringungen oder von einzelnen Gewalttaten von Geflüchteten ${ }^{9}$ gegenüber Frauen dazu genutzt, Gewalt gegen Menschen, die sich auf der Flucht befinden, zu legitimieren und ihre Kriminalisierung fortzuschreiben.

Um uns diesen schwierigen Fragestellungen zu stellen, werfen wir einen kurzen Blick auf die Diskurse im Zusammenhang von (post-)kolonialer Fluchtmigration und muslimischen Fluchtmigrant_innen in Europa. Dabei wird versucht die Kontinuitätslinien freizulegen, die seit der imperialen Herrschaft Europas die ideologische Instrumentalisierung der Gewalt an Frauen, die innerhalb (post-)kolonialer und fluchtmigrantischer Communities stattfindet, transparent werden lässt. Wir fragen uns: Wer stimmt dem essenzialisierenden Reden von dem Islam oder den muslimischen Geflüchteten zu? Welche Funktion erfüllen die öffentlichen Diffamierungen von Menschen, die auf der Flucht sind? Und welche Rolle spielen hier Geschlechterdynamiken? Wir nehmen dabei sowohl die pauschalisierenden Repräsentationen des Islams und der Muslime und Muslima in den Blick als auch Vorstellungen von den Afrikanern und der afrikanischen Kultur, die per se als frauenverachtend beschrieben wird. In On the Postcolony (2001, S. 39 ff.) hat Achilles Mbembe überzeugend herausgearbeitet, welche Rolle die Repräsentation des afrikanischen Kontinents als „Leere“ für die europäische Selbstwahrnehmung hat. Dass innerhalb dieser und ähnlicher Diskurse die Geschlechterdynamiken eine wichtige Rolle spielen, lässt sich dagegen in den Schriften feministisch-postkolonialer Autor_innen nachlesen (etwa McClintock 1995).

Als gewalttätiges Zusammentreffen westlicher mit präkolonialen patriarchalischen Hegemonien kann Kolonialismus ohne eine Theorie der Geschlechterregimes kaum verstanden werden (vgl. Spivak 1994). Die postkoloniale Kritikerin McClintock (1995) argumentiert darum sehr richtig, dass die Erfahrungen mit Rassismus, Sexismus und Klassenunterdrückung nicht unabhängigen Bereichen zugeordnet werden können. Vielmehr stehen diese in einer gegenseitigen - manchmal widersprüchlichen und konfliktgeladenen - Beziehung zueinander (ebd., S. 5). Gemäß dieser Vorstellung sind ,Rasse“ und Geschlecht nicht primär eine Frage von Hautfarbe und Sexualität, sondern ebenso eine von unterworfener Arbeit und imperialer Ausplünderung (ebd., S. 6). Imperialismus kann für McClintock ohne eine Theorie der Geschlechterregime auch deswegen schwerlich verstanden werden, weil die Mehrheit kolonisierter Frauen bereits vor der Kolonisierung eine prekäre soziale Position innehatte. Eine Tatsache, die ihrer kolonialen Ausbeutung einen anderen Charakter verlieh, als dies für die kolonisierten Männer der Fall war. Geschlechterdy-

\footnotetext{
${ }^{9}$ Geschlechtergewalt in den Erstunterbringungen von Geflüchteten ist eine nicht zu verleugnende Tatsache. Zumeist wird hier weggesehen und argumentiert, die Geflüchteten hätten zurzeit wichtigere Probleme. Für die Frauen bedeutet dies, dass sie kaum Unterstützung erhalten und mithin der Gewalt ausgeliefert sind.
} 
namiken - wie auch Sexualitätspolitiken - erwiesen sich als fundamental wichtig, um die imperialen Herrschaftsverhältnisse zu sichern. Kaum zufällig wurde auf beiden Seiten der kolonialen Grenzziehung der weibliche Körper zur Markierung kultureller Differenz instrumentalisiert (vgl. Castro Varela und Dhawan 2015, S. 47 ff.). Die tatsächliche Geschlechtergewalt innerhalb kolonisierter Gesellschaften lieferte den Europäer_innen eine unentbehrliche Legitimation für die koloniale Gewaltherrschaft. Die imperialen Herrscher plädierten für eine ,zivilisatorische Reformbewegung" (Spivak 1994), die die koloniale Mission als geradezu unweigerliches Ereignis charakterisierte (vgl. genauer Castro Varela und Dhawan 2015, S. 193 ff.). Zudem vermittelte die koloniale Ideologie dem europäischen Mann, dass er es sei, der die Bürde zu tragen habe, dem ,Rest der Welt' die zivilisatorischen Errungenschaften Europas zu überbringen (Spivak 1994), - wenn es sein müsse mit Gewalt. Zu diesen Errungenschaften wurde immer auch die Geschlechtergerechtigkeit gezählt. Doch wissen wir um die Kämpfe, die in Europa eben zu jenen imperialen Zeiten ${ }^{10}$ geführt wurden: Im 19. Jahrhundert forderten Frauen einerseits das Wahlrecht und ein umfassendes Recht auf Bildung und hatten noch für viele Jahre nicht denselben Zugang zu öffentlichen Räumen wie weiße europäische Männer. Auf der anderen Seite interpretierten die antikolonialen nationalistischen Bewegungen Interventionen in Richtung mehr Geschlechtergerechtigkeit als kolonialistischen Eingriff und reagierten darauf mit eigenen Neuordnungen des Geschlechterregimes. Sie fürchteten und beklagten die Verwestlichung der ,eigenen Frauen', während ihnen ein gewisser Grad der Imitation des Westen für die Männer als unvermeidbar galt. Der indigenen Frau wurde im antikolonialen nationalistischen Diskurs durchaus kulturelle Überlegenheit zuerkannt, doch die Ehre der respektablen Frau verlangte dennoch nach Verteidigung (etwa Sangari und Vaid 1989). Der westliche Feminismus wurde von den antikolonialen Nationalisten geradezu systematisch verteufelt, galt es doch die Grenzziehungen zwischen den westlichen und den indigenen Frauen zu erhalten. Darüber hinaus nutzten die verschiedenen religiösen Institutionen den Körper der Frau als Schlachtfeld in ihrem Kampf um Erlangung institutioneller Macht (vgl. Jayawardena und de Alwis 1996, S. ix; van der Veer 2001, S. 96 ff.). Die Überschneidung von religiösem Nationalismus und patriarchaler Gewalt ist Bestandteil aller sogenannten Fundamentalismen, ganz gleich ob es sich dabei um Hinduismus, Buddhismus, Islam, Sikhismus oder das Christentum handelt. Gleichzeitig darf nicht vergessen werden, dass religiöse Bewegungen äußerst machtvolle Oppositionsbewegungen gegen die koloniale Herrschaft darstellten. Dies ist geradezu symptomatisch für ihre ambivalente Position. Im anti-imperialen Widerstand konstruierten diese eine nationale Identität und ein kulturelles Bewusstsein als wirkungsmächtige Alternative zu der aufoktroyierten Identität und Kultur der europäischen Herrschaft. Doch genau diese effektvolle antikoloniale Waffe hatte für das Leben von Frauen in den (ehemaligen) Kolonien schwerwiegende Folgen. Das nationalistische Projekt setzte eine symbolische Ordnung kultureller Reinheit durch, während Frauen gleichsam zum Eigentum des männlich-nationalen Kollektivs erklärt wurden, welches vor den moralisch verdorbenen westlichen Einflüssen geschützt werden musste. Aus diesem Grunde wurden juristische und reformerische Transformationen als Einmischung der

10 Mitte des 19. Jahrhunderts gilt als die Hochzeit der imperialen europäischen Herrschaft. 
(kolonialen) Regierungen in den heiligen Raum der Familie interpretiert und zum Widerstand gegen diese aufgerufen (Jayawardena und de Alwis 1996, S. xi). Für Frauen bedeutete dies fortan, eine äußerst schwierige Position zwischen Geschlecht und religiöser Identität besetzen zu müssen. Dieses Dilemma hatte zur Folge, dass einige Frauen den Kampf für die eigene kulturelle und religiöse Identität über den Kampf um Geschlechtergerechtigkeit stellten (ebd., S. xiii). Eben weil das nationalistische Projekt auf die religiöse und ethnische Identität beschränkt blieb, wurde die den Frauen zugewiesene Rolle der Zuständigkeit für die Reinhaltung der nationalen Identität schicksalsträchtig (ebd.). Aus diesem Grunde ist die Dekonstruktion religiöser und ethnischer Identitäten ein entschieden politisches Projekt, denn die Thematisierung von Geschlechtergewalt stellt eine wichtige Strategie dar, um die illusionäre Natur religiöser nationaler Identitäten freizulegen (vgl. ebd., S. Xv). So ist es möglich, stereotype Vorstellungen von als monolithisch beschriebene Religionen und homogene Gemeinschaften über ein nuanciertes Lesen der Verortung muslimischer Frauen innerhalb ihrer religiösen und ethnischen Community wie auch in ihrem Verhältnis zu Recht, Staat und Ökonomie in ihrer Fragmentierung offenzulegen (vgl. ebd.). Die Ablehnung von Frauenrechten durch heutige religiöse Führer legitimiert nicht nur ein repressives patriarchales Regime, sondern spielt auch den Interessen einiger (postkolonialer) Staaten und diasporischer Communities in die Hände, die nach wie vor das Ziel verfolgen, eine singuläre Identität zu formen (vgl. ebd., S. xvi). Darüber hinausgehend zeigen die sexuellen und moralischen Kodierungen, die Frauen über hegemoniale patriarchale Institutionen und Instrumente wie Staat, Recht, Religion, Familie, Community etc. aufgezwungen wurden, entscheidende Ähnlichkeiten. So sind es beispielsweise immer die weiblichen Mitglieder, die dazu verpflichtet werden, die angeblichen Traditionen ethnisierter Gruppen zu schützen (vgl. etwa Coomaraswamy 2005). Die Erfindung der Traditionen hat hier einen repressiv-funktionalen Charakter.

Bei der Analyse genderspezifischer Unterdrückungsformen scheint es geboten, die historischen Verbindungen in den Blick zu nehmen, da es diese Verflechtungen sind - ein Erbe kolonialer Herrschaftstaktiken -, die jeden Kampf um Geschlechtergerechtigkeit von Frauen im globalen Süden wie auch in fluchtmigrantischen und diasporischen Kollektiven mit dem westlichen Imperialismus beziehungsweise hegemonialen Kräften verbindet (vgl. Castro Varela und Dhawan 2015, S. 163 ff.). Daneben ist es notwendig, die aktuellen sozialen Positionierungen von Frauen historisierend $\mathrm{zu}$ betrachten, so dass transparent wird, dass Praxen, die einst für die Handlungsmächtigkeit derselben standen, häufig im Prozess der Kolonisierung in unterdrückerische transformiert wurden (vgl. etwa Oldenburg 2002).

Heute sind die postkolonialen Regierungen überwältigend männlich, während die physische Gewalt gegen Frauen in den postkolonialen Räumen zu- und nicht abnimmt (siehe etwa Dhawan 2013b). Saskia Sassen hat in diesem Zusammenhang den scharfen Begriff ,feminization of survival“ (2000) geprägt, der darauf verweist, dass die Mehrheit der weltweit sich auf der Flucht befindenden Millionen von Menschen weiblich ist und dass es vor allem Frauen sind, die die gewaltigen Kosten der Konflikte, Kriege, des Klimawandels und der neoliberalen Globalisierung zu tragen haben. 
Das koloniale Erbe beeinflusst selbst die soziale Positionierung von Frauen im globalen Norden und rahmt die Möglichkeiten ihrer Handlungsmacht. Die differenzierten postkolonialen Analysen, so unsere Annahme, zeigen sich für die Beschreibung der diffizilen Situation fluchtmigrantischer Frauen als durchaus ertragreich.

\section{Rassismus - Sexismus und die vertrackte Situation der Fluchtmigrantin}

Aihwa Ong zufolge kämpfen muslimische Feministinnen heute weltweit sowohl gegen ein islamisches Patriarchat als auch gegen einen kulturellen und ökonomischen Imperialismus (vgl. Ong 1999, S. 355). Der Repräsentation als die kulturell Anderen, als rückständig und prämodern, versuchen islamische Autoritäten unter anderem dadurch zu begegnen, dass sie religiöse Moralvorstellungen durchsetzen, die erneut Frauen unterwerfen. Ein bekanntes Muster, denn wenn Männer als kulturell minderwertig konstruiert werden, so Ong, begegnen sie der Unterwerfung und Demütigung nicht selten mit einer Retraditionalisierung der Frauen zugewiesenen Rollen. Es wird dann nach einer Belebung angeblich traditioneller Werte gerufen und die Abschirmung gegen Außeneinflüsse vorangetrieben (ebd.). In den letzten Jahrzehnten hat Ong zufolge der islamische Diskurs an vielen postkolonialen Orten - wie auch in der Diaspora - ein islamisch-männliches Ethos artikuliert, das Frauen in die häusliche Sphäre zurückband (vgl. ebd., S. 357). Ong führt hier das Beispiel der malaiischen Debatte um Gewalt in der Ehe an. Schon die Idee wurde in Malaysia von der konservativen Mehrheit als westlich beschrieben und so die Diskussion bereits auf Begriffsebene als kulturell unangemessen abgelehnt (vgl. ebd., S. 367). Beispielhaft zitiert sie in diesem Zusammenhang den Vorsitzenden der malaiischen Anwaltskammer, der bemerkte: „We should not blindly import concepts unsuitable for our cultural background and values“ (ebd.). Es ist dies ein gutes Beispiel für die recht übliche Taktik, die Erfolge lokaler feministischer Bewegungen mit einer Diskussion um westliche Werte zu diskreditieren.

Diese Praxis hat unweigerliche Auswirkungen auf Dynamiken in der Diaspora. So spricht die Rechtsanwältin Sara Hossain, die sich extensiv mit Zwangsehen innerhalb diasporischer Communities beschäftigt hat (vgl. Hossain 2005, S. 384), von einem postkolonialen Paternalismus, der die Fluchtmigrantin als Opfer positioniert und gleichzeitig fluchtmigrantische Communities dämonisiert. Mit der Folge, dass sexistische Gewalt innerhalb fluchtmigrantischer Räume (siehe etwa IWSPACE 2015) den angeblich wichtigeren Kämpfen gegen Rassismus untergeordnet wird. Es scheint uns dringlich, diese verzwickte Lage zu beleuchten, da ansonsten die Frage nach Geschlechtergewalt zwischen Rassismus und Kulturrelativismus unterzugehen droht oder nur von denjenigen auf die politische Agenda gesetzt wird, die sie zur Instrumentalisierung eigener Herrschaftsinteressen $\mathrm{zu}$ funktionalisieren wissen.

Ähnlich der Selbstdarstellung der Kolonialherren, die behaupteten, dass sie die unterdrückte kolonisierte Frau retten müssten, ist es Teil der dominanten Selbstrepräsentation Europas, sich als die rettende Instanz der ,hilfsbedürftigen Fluchtmigrantin“ zu verstehen. Die stereotypen Repräsentationen zeigen etwa muslimische Frauen als Opfer ihrer Religion und Kultur. Die handlungsmächtige Muslima scheint 
dagegen nicht darstellbar zu sein. Dabei wird weiter an einer asymmetrischen Ignoranz festgehalten, die nicht willens ist, sich der Komplexität des Islams zu stellen, dessen Praxen sich deutlich nach historischen, regionalen und ethnischen, aber auch klassenspezifischen Kontexten unterscheiden (siehe etwa Said 1978). In den westlichen Medien finden wir dagegen fast durchgehend eine dekontextualisierte Darstellung des Islams als barbarisch, frauenfeindlich und homophob (vgl. Said 1997; Dhawan 2013a). Lila Abu-Lughod (2013) dagegen verwehrt sich gegen eine Homogenisierung der muslimischen Frau und ihrer Konstruktion als Opfer des Islams. Dies, so Abu-Lughod, würde schließlich nur dem Westen nutzen, der seine kolonialen Rettungsfantasien weiter ausleben und dadurch Interventionen unterschiedlicher Art - auch kriegerischer - legitimieren kann. Gleichzeitig werden die komplexen Machtkonfigurationen, die das Handlungspotential muslimischer Frauen einschränken, rigoros verkannt. Dabei darf nicht unerwähnt bleiben, dass das Bild der handlungsohnmächtigen Fluchtmigrantin im Allgemeinen und der muslimischen Frau im Besonderen auch von einem westlichen säkularen Feminismus gestützt wird (etwa Mohanty 1988, S. 151). Shahnaz Khan (2005, S. 310) bemerkt, dass antikoloniale und antiwestliche Bewegungen nicht nur durch eine Zunahme sozialer und sexueller Kontrolle von Frauen charakterisierbar sind, sondern sie dies regelrecht zum Mittelpunkt ihrer identitätspolitischen Agenda erheben. Wenn Frauen, die durch den orientalistischen Diskurs entfremdet wurden, sich auf der Suche nach Identität dem Islam zuwenden, so sehen sie sich nicht selten mit islamistischen Versuchen konfrontiert, ihre Körper in eine rigide sexistische Struktur einzupassen. Orientalismus und Islamismus bilden gewissermaßen zwei Seiten einer Medaille: Im Ergebnis wird die muslimische Frau ihrer Selbstbestimmung beraubt und in ihrer Selbstrepräsentation eingeschränkt. In der Migration, auf der Flucht und im Exil sieht sie sich Rassifizierungsprozessen gegenüber, während sie nicht selten einer rigiden sozialen und sexuellen Kontrolle durch die eigene ethnisch-religiöse Community unterworfen wird (ebd., S. 333). Das Muslimisch-Sein der Frauen innerhalb einer marginalisierten sozialen Gruppe, so Khan, ist signifikant fragiler als in den Herkunftsländern (ebd., S. 312). Während die Integration als ein Schlüsselprozess für Erfolg beschrieben wird (kritisch hierzu Castro Varela 2013), müssen fluchtmigrantische Frauen gleichzeitig ihre Positionierungen innerhalb der eigenen Community aushandeln. Diskurse, die fluchtmigrantische Kollektive als statisch und monolithisch wahrnehmen, schränken den Handlungsradius der weiblichen Subjekte stark ein. Wenn sexistischen Angriffen innerhalb und außerhalb der eigenen Community widerstanden wird, so kann dieser Widerstand der minorisierten Gruppe als Ganzes schaden. So werden fluchtmigrantische Frauen letztlich in einer Gesellschaft verletzlich zurückgelassen, in der sie nicht nur Sexismus, sondern auch Rassismus bewältigen müssen (ebd., S. 313). Muslimische Migrantinnen, so Khan, werden erst in das Kollektiv der Anderen verbannt und dann entwertet, weil sie eben diesem Kollektiv angehören (ebd., S. 314). Patriarchale, ahistorische und romantisierende Betrachtungen der ,eigenen Kultur verneinen dagegen gleichsam die Dynamik von Identitätsverhandlungen innerhalb von migrantischen Communities. Obendrein tendiert der dominante Mediendiskurs dazu, den Dualismus zwischen den „Unterdrückten“ und den „Emanzipierten“ zu verstärken, sodass zum Beispiel die orientalistische Perspektive auf das Kopftuch als unterdrückend der islamistischen Perspektive desselben als befreiend gegenübersteht 
(Khan 2005, S. 314). Die Suche nach Strategien gegen sexistische und rassistische Gewalt gerät zu einer schwierigen und komplexen Praxis.

\section{Fluchtmigration und sexualisierte Gewalt}

Migration und Flucht sind geradezu zu Symbolen globaler Ungleichheiten geworden. Die Möglichkeiten für Menschen auf der Flucht, auf legalem Wege in den globalen Norden einzuwandern, werden immer weniger. Europa schottet sich ab: Routen werden durch Zäune und Mauern versperrt, die Einwanderungsgesetze überall verschärft, Quoten eingeführt, zwischenstaatliche Deals geschlossen, die nur die angebliche Sicherheit der eigenen Bürger_innen im Fokus haben, und das gesamte administrative Procedere der Legalisierung: Registrierung, Antragstellung, Zuweisung etc. wird mehr und mehr kafkaesk. Mobilität wird zunehmend illegalisiert, doch hindert dies die Menschen nicht daran, weiterhin vor Verfolgung, Elend und Krieg zu fliehen. Die extra-legale Grenzüberschreitung erweist sich dabei für Frauen (wie auch Queers) auch aufgrund sexualisierter Gewalt und geschlechtsspezifischer Verletzlichkeiten als signifikant schwerwiegender. Die europäischen Nationalstaaten allerdings betonen die Verletzbarkeit ihrer Grenzen und haben einen Diskurs der Angst vis-à-vis der Fluchtmigration etabliert, der die zunehmende Ausgrenzung und Gewalt gegenüber Fluchtmigrant_innen legitimiert.

In der Sylvesternacht 2015/2016 kam es in Köln und Hamburg zu sexuellen Übergriffen auf Frauen. Unterschiedliche Gruppen junger Männer, die als nicht-deutsch - manchesmal als arabisch - beschrieben wurden, haben Frauen sexuell belästigt und bestohlen. Die Zahlen variieren stark, aber die Rede ist von einer dreistelligen Zahl und mithin von schweren Vorfällen, auf die international medial reagiert wurde. Spannenderweise geht es in den Debatten um die Sylvesternacht jedoch weniger um die tatsächliche sexualisierte Gewalt: eher wird die Herkunft der Täter thematisiert. Und so geht es in den Debatten kaum um Formen und Strategien des Opferschutzes, sondern um die Verschärfung des Asylrechts und um die Forderung nach einer erleichterten Abschiebung von jungen muslimischen Geflüchteten. Sexismus wird zu einem Problem männlicher muslimischer Subjekte, und das, obwohl bekannt ist, dass Sexismus in europäischen Städten ubiquitär ist. Die Fokussierung auf die vermeintlich arabische Herkunft der Täter in den Berichterstattungen vernachlässigt darüber hinaus, dass nach wie vor die meisten sexuellen Übergriffe von Verwandten und (Ex-)Partnern ausgehen und dass Women of Color in Europa nicht nur jeden Tag sexualisierte Übergriffe erleben, sondern dass rassistische Praxen geradezu zu ihrem Alltag gehören. Weswegen die Soziologin Kira Kosnick (2016, S. 2) zu Recht bemerkt, dass zum einen, ,über die Funktion und Bedeutung von sexualisierter Gewalt und strukturellem Sexismus gesprochen werden sollte" und dass zum anderen die Diskussion erst komplett und sinnvoll wäre, wenn auch über Rassismus und die andauernde Gewalt gegen Geflüchtete gesprochen würde. Die tausenden Übergriffe auf Geflüchtete und die grausamen Angriffe auf die Unterkünfte von geflüchteten Menschen, die in Deutschland und Österreich Schutz suchen, sind schließlich mehr als beängstigend. Darüber hinaus zeigen die Diskurse rund um die sexualisierte Gewalt, die nur Geflüchteten zugeschrieben wird, wie schnell die Mehrheit nicht nur 
wieder Rassismus salonfähig macht, sondern auch listig feministische Forderungen instrumentalisiert, um rassistische Diskurse zu legitimieren. Die Bildungswissenschaftlerin Astrid Messerschmidt (2016, S. 1) weist zudem auf eine andere Funktion dieser spezifischen Thematisierung hin. ,(D)ie Ereignisse“, so Messerschmidt, „,bieten eine Gelegenheit, um etwas anderes zu tun: nämlich den nationalen Innenraum als unschuldig zu repräsentieren. Unschuld ist eine Obsession in der Bundesrepublik Deutschland, Ausdruck einer postnationalsozialistischen Resonanz.“

Die Täter von Köln haben nicht nur alle Männer, die als anders markiert werden, diskreditiert, sie befreien auch die europäischen Männern von dem Verdacht des Sexismus und Rassismus. „Die Skandalisierung der Straftaten in der Silvesternacht als Integrationsproblematik ist ein erschreckendes Beispiel für die Kulturalisierung des politischen Diskurses“ (Kosnick 2016, S. 2). Ein statisches und essentialistisches Kulturverständnis muss in einer Situation, in der die patriarchale Unterdrückung von Frauen als unveränderliches Merkmal des Islams oder auch einer ,arabischen Herkunftskultur' betrachtet wird, fatale Konsequenzen zeitigen - das umso mehr, wenn die Reinheits- und Unschuldsobsession deutscher und österreichischer Mehrheitsangehöriger mitbedacht wird. Kultur und Religion können nicht, wie Kosnick richtig schreibt, ,isoliert von geopolitischen Machtverhältnissen, globaler sozialer Ungleichheit und kolonialer Geschichte diskutiert werden“" (ebd.).

Positiv sind deswegen die erstarkenden feministischen Widerstände, die Feminismus immer als gleichzeitig antisexistisch und antirassistisch ${ }^{11}$ beschreiben. Diese verdeutlichen, dass die zum Teil scharfen Debatten in der weißen Frauenbewegung um Rassismus, Klassismus und Antisemitismus nicht einfach verpufft sind.

\section{Die Fluchtmigrantin und ihre dilemmatische Position vis-à-vis dem (europäischen) Staat}

Eine selektive, sehr spezifische Positionierung gegenüber dem nicht-westlichen Feminismus scheint in der Mehrheitsbevölkerung die Regel zu sein. So ist es nur auf den ersten Blick paradox, dass etwa das Interesse an Frauen wie Malala Yousafzai, die jüngste Preisträgerin in der Geschichte des Nobelpreises, mit anti-muslimischen Politiken einhergeht. Die westliche Porträtierung dieser Frauen folgt dabei einem immer gleichen Schema: Sie sind die einsamen, starken Streiterinnen im Kampf gegen das Patriarchat ihrer Kultur und Religion. Selten werden sie dagegen als Teil großer sozialer und politischer Bewegungen gesehen, die in diesen Ländern für mehr Freiheit und Demokratie kämpfen (vgl. Amireh 2005, S. 269; auch Mernissi 1992). Es kann also kaum verwundern, dass problematische Bücher wie zum Beispiel jene von Necla Kelek oder Seyran Ateş weiterhin Bestseller sind. Die Politik der Repräsentation tappt hier gewissermaßen in eine bekannte Falle: Die authentische Stimme spricht und sagt das, was die Mehrheit hören will. In diesem Moment verquickt sich ein hegemoniales Zuhören, welches nur das hört, was die dominanten Verhältnisse reflektiert, mit der Forderung der politisierten Minderheiten nach dem Recht auf eine eigene, eben authentische Stimme.

11 https://reclaimfeminism.org/. 
In den letzten Jahrzehnten hat sich im Westen geradezu ein Fluchtmigrant_innenRettungsdiskurs etabliert, der gewinnbringend vermarktet wird, indem in Büchern, Talkshows, wissenschaftlichen Arbeiten etc. exakt die Bilder geliefert werden, die die Mehrheit sehen will. Dies erinnert uns an eine lange voyeuristische Tradition im Westen, die einer Nostalgie nach der ganz Anderen frönt, die auf ihre Rettung durch den Westen harrt. Gleichzeitig ist zu bezweifeln, dass die Verharmlosung und Negierung von Gewalt innerhalb fluchtmigrantischer und diasporischer Communities eine gute Idee ist. Die fatalen Effekte einer solchermaßen einseitigen Strategie für das Leben rassifizierter Frauen sind aus den nationalen Unabhängigkeitskämpfen ehemaliger Kolonien wohl bekannt. Sie schützen die Communities im Allgemeinen, gefährden aber das Leben minorisierter Frauen (und auch anderer Minderheiten innerhalb der Minderheiten).

Wie wir gezeigt haben, ist im Westen die Strategie der Legitimierung von Dominanz durch das Motiv der notwendigen Befreiung der anderen Frau nicht neu, weswegen die aktuellen Debatten um Migration und Integration nicht zufällig deutlich koloniale Züge zeigen, die unter anderem rassistische Praxen legitimieren.

Die Verschärfung des Migrationsrechts, brutale Grenzziehungen und die immer stärkere Kontrolle von Fluchtmigrant_innen wird unter anderem damit begründet. Der Ruf nach mehr Staat, der Produkt dieses Diskurses ist, geht einher mit Forderungen nach erleichterten Abschiebungen, Erschwerung der Einreise etc. Schließlich ist er Teil eines Bedrohungsszenarios, welches Flucht und Migration schlichtweg zum Sicherheitsrisiko erklärt, auf das mit mehr staatlicher Kontrolle reagiert werden müsse (vgl. Castro Varela 2005; siehe auch Brown 1992).

Im Kontext von antiislamischem Rassismus, eines zunehmenden religiösen Fundamentalismus und der anhaltenden Gewalt gegen Frauen ist es dringend geboten, eine Perspektive einzunehmen, die sich auf die Überschneidungen unterschiedlicher Machtachsen konzentriert. Doch muss gleichzeitig auch der Interessenkonflikt innerhalb migrantischer Kollektive für eine Analyse der Gewalt gegen Fluchtmigrant_innen grundlegend sein.

\section{Schluss}

Integrationspolitiken versuchen Migrant_innen und Geflüchtete in eine imaginierte Gemeinschaft einzufügen. Fokussieren wir die weiblichen Mitglieder fluchtmigrantischer und diasporischer Communities aus einer feministisch-postkolonialen Perspektive, so wird ein Dilemma sichtbar, welches für die Instrumentalisierung von Migrantinnen und Geflüchteten verantwortlich zeichnet. Ein wachsender antimuslimischer Rassismus, der sich in Europa immer wieder gewalttätig Ausdruck verschafft, scheint eine adäquate Artikulation sexistischer Gewalt innerhalb muslimisch-fluchtmigrantischer Communities - oder von fluchtmigrantischen Männern ausgehend - unmöglich zu machen. Werden sexistische Vorfälle hier skandalisiert, führt dies unweigerlich zur Bestätigung rassistischer und orientalistischer Vorurteile, die rassistische Alltagspraxen und die Verschärfung von Asyl- und Migrationspolitiken zu legitimieren scheinen. Eine gute Lösung scheint es nicht zu geben, und dennoch muss beständig beides gemacht werden: das Offenlegen rassistischer Pra- 
xen und die Thematisierung von Gewalt gegen Frauen (und anderer verletzlicher Subjekte) innerhalb fluchtmigrantischer und diasporischer Communities. Dies erfordert ein hohes Maß an dekonstruktiver Wachsamkeit und ein politisch-strategisches Nachdenken, welches auch ein Nachdenken über die Rolle, die der Staat in diesem Dilemma einnimmt, beinhaltet. Auch hier gilt es, eine feministische Politik zu etablieren, die das Unmögliche wagt, um Dekolonisierung und Demokratisierung voranzutreiben. Die Auseinandersetzung mit den Dilemmas und Aporien scheint der einzige gangbare Weg zu sein.

Open access funding provided by University of Innsbruck and Medical University of Innsbruck.

Open Access Dieser Artikel wird unter der Creative Commons Namensnennung 4.0 International Lizenz (http://creativecommons.org/licenses/by/4.0/deed.de) veröffentlicht, welche die Nutzung, Vervielfältigung, Bearbeitung, Verbreitung und Wiedergabe in jeglichem Medium und Format erlaubt, sofern Sie den/die ursprünglichen Autor(en) und die Quelle ordnungsgemäß nennen, einen Link zur Creative Commons Lizenz beifügen und angeben, ob Änderungen vorgenommen wurden.

\section{Literatur}

Abu-Lughod, Lila. 2013. Do Muslim Women Need Saving? Cambridge: Harvard University Press.

Amireh, Amal. 2005. Framing Nawal El Saadawi: Arab Feminism in a Transnational World. In Gender, Politics and Islam, Hrsg. Therese Saliba, Allen Carolyn, und Judith Howard, 269-304. New Delhi: Orient Longman.

Brown, Wendy. 1992. Finding the Man in the State. Feminist Studies 18(1):7-34.

Butler, Judith. 1999. Gender trouble: Feminism and the Subversion of Identity. London: Routledge.

Butler, Judith. 2004. Precarious Life: The Powers of Mourning and Violence. London: Verso.

Castro Varela, María do Mar, und Nikita Dhawan. 2015. Postkoloniale Theorie. Eine kritische Einführung, 2. Aufl., Bielefeld: transcript.

Castro Varela, María do Mar. 2005. Integrationsregime und Gouvernementalität. Herausforderungen an interkulturelle/internationale soziale Arbeit. nеие praxis 8:152-164.

Castro Varela, María do Mar. 2013. Ist Integration nötig? Eine Streitschrift. Freiburg i. Br.: Lambertus.

Castro Varela, María do Mar. 2015. Von der Notwendigkeit eines epistemischen Wandels. Postkoloniale Betrachtungen auf Bildungsprozesse. In Migration: Auflösungen und Grenzziehungen. Perspektiven einer erziehungswissenschaftlichen Migrationsforschung, Hrsg. Thomas Geier, und Katrin U. Zaborowski, 43-59. Wiesbaden: Springer.

Coomaraswamy, Radhika. 2005. Identity Within: Cultural Relativism, Minority Rights and the Empowerment of Women. In Men's Laws, Women's Lives. A Constitutional Perspective on Religion, Common Law and Culture in South Asia, Hrsg. Indira Jaising, 23-55. New Delhi: Kali for Women.

Daly, Mary. 1978. Gyn/Ecology: The Metaethics of Radical Feminism. Boston: Beacon Press.

Dhawan, Nikita. 2013a. The empire prays back: Religion, secularity and queer critique. Boundary 2 40(1):191-222.

Dhawan, Nikita. 2013b. Postkoloniale Gouvernementalität und die Politik der Vergewaltigung. Gewalt, Verletzlichkeit und der Staat. Femina Politica. Zeitschrift für Feministische Politikwissenschaft 22(2):85-104.

Dhawan, Nikita. 2015. Homonationalismus und Staatsphobie: Queering Dekolonisierungspolitiken, Queer-Politiken dekolonisieren. Femina Politica. Zeitschrift für feministische Politikwissenschaft: Perspektiven Queerfeministischer Politischer Theorie 24(1):38-51.

Galtung, Johan. 1988. Strukturelle Gewalt. Beiträge zur Friedens- und Konfliktforschung. Reinbek bei Hamburg: Rowohlt.

Gemende, Marion. 2007. Eva ist emanzipiert, Mehmet ist ein Macho. Zuschreibung, Ausgrenzung, Lebensbewältigung und Handlungsansätze im Kontext von Geschlecht und Migration. Weinheim, München: Juventa.

Hill Collins, Patricia. 1990. Black feminist thought: knowledge, consciousness, and the politics of empowerment. New York, London: Routledge. 
Hossain, Sara. 2005. The Right to Choose: Whether, When and Whom to Marry. In Men's Laws, Women's Lives. A Constitutional Perspective on Religion, Common Law and Culture in South Asia, Hrsg. Indira Jaising, 374-389. New Delhi: Kali for Women.

IWSPACE. 2015. In our own Words. Refugee Women in Germany tell their stories. Berlin: Selbstverlag.

Jayawardena, Kumari, und Malathi de Alwis. 1996. Introduction. Communalising women's sexuality in South Asia. In Embodied Violence. Communalising Women's Sexuality in South Asia, Hrsg. Kumari Jayawardena, und Malathi de Alwis, ix-xxiv. New Delhi: Kali for Women.

Khan, Shahnaz. 2005. Muslim Women: Negotiations in the Third Space, Gender, Politics and Islam. In Gender, Politics and Islam, Hrsg. Therese Saliba, Allen Carolyn, und Judith Howard, 305-336. New Delhi: Orient Longman.

Kosnick, Kira. 2016. Köln und die Folgen. Unireport Goethe-Universität Frankfurt am Main 49/1. http:// www.unireport.info/59936941/Unireport_1-16.pdf. Zugegriffen: 09. Apr 2016.

Lorde, Audre. 1984. An Open Letter to Mary Daly. Erstausgabe 1979. www.historyisaweapon.com/ defcon1/lordeopenlettertomarydaly.html. Zugegriffen: 09. Apr 2016.

Lorde, Audre. 1984. Sister Outsider: Essays and Speeches. Portland: The Crossing.

Mamozai, Martha. 1982. Herrenmenschen. Frauen im deutschen Kolonialismus. Reinbek bei Hamburg: Rowohlt.

Mbembe, Achille. 2001. On the Postcolony. Berkeley, Los Angeles, London: University of California Press.

McClintock, Anne. 1995. Imperial Leather: Race, Gender and Sexuality in the Colonial Contest. New York, London: Routledge.

Mernissi, Fatima. 1992. Die Angst vor der Moderne. Frauen und Männer zwischen Islam und Demokratie. Hamburg, Zürich: Luchterhand.

Messerschmidt, Astrid. 2016. Nach Köln - sprechen über Sexismus und Rassismus. http://www. rassismuskritik-bw.de/nach-koeln-sprechen-ueber-sexismus-und-rassismus/. Zugegriffen: 09. Apr 2016.

Mohanty, Chandra Talpade. 1988. Aus westlicher Sicht: feministische Theorie und koloniale Diskurse. beiträge zur feministischen theorie und praxis 23:149-162.

Oldenburg, Veena T. et al. 2002. Dowry Murder. The Imperial Origins of a Cultural Crime. Oxford: Oxford University Press.

Ong, Aihwa. 1999. Muslim Feminism: Citizenship in the shelter of corporatist Islam. Citizenship Studies 3(3):355-371.

Republik Österreich/Bundesministerium für Inneres. 2016. Refugee-Guide. http://www.refugee-guide.at/ start.html. Zugegriffen: 07. Apr 2016.

Said, Edward. 1978. Orientalism. New York: Vintage.

Said, Edward. 1997. Covering Islam. How the Media and the Experts Determine how we see the Rest of the World. New York: Vintage.

Sangari, Kumkum, und Sudesh Vaid (Hrsg.). 1989. Recasting Women. Essays in Colonial History. New Delhi: Kali for Women.

Sassen, Saskia. 2000. Countergeographies of globalization: The feminization of survival. Journal of International Affairs 53(2):503-524.

Spivak, Gayatri C. 1994. Can the Subaltern Speak? In Colonial Discourse and Post-Colonial Theory: A Reader, Hrsg. Patrick Williams, und Laura Chrisman, 66-111. Hemel Hemstead: Harvester Wheatsheaf.

Thürmer-Rohr, Christina (Hrsg.). 1989. Mittäterschaft und Entdeckungslust. Studienschwerpunkt ,Frauenforschung ' am Institut für Sozialpädagogik der TU Berlin. Berlin: Orlanda.

van der Veer, Peter. 2001. Imperial Encounters: Religion and Modernity in India and Britain. Princeton: Princeton University Press.

María do Mar Castro Varela Prof. ${ }^{\text {in }}$ Dr. ${ }^{\text {in }}$, ist Professorin für Allgemeine Pädagogik und Soziale Arbeit, Alice Salomon Hochschule, Berlin, und derzeit Visiting Fellow am Institut für die Wissenschaften vom Menschen (IWM), Wien. Aktuelle Publikationen: Homosexualität_en und Alter(n), Wiesbaden: Springer_VS Verlag (2016); mit Nikita Dhawan: Postkoloniale Theorie. Eine kritische Einführung, 2. komplett überarbeitete und erweiterte Auflage, Bielefeld: Transcript (2015); Ist Integration nötig? Eine Streitschrift, Freiburg i. Br.: Lambertus (2013). 
Nikita Dhawan Univ.-Prof. ${ }^{\text {in }}$ Dr. $^{\text {in }}$, ist Professorin für Politische Theorie mit thematischer Akzentuierung im Feld der Frauen- und Geschlechterforschung sowie Direktorin der Interfakultären Forschungsplattform Geschlechterforschung: Identitäten - Diskurse - Transformationen an der Universität Innsbruck. Aktuelle Publikationen: Negotiating Normativity. Postcolonial Appropriations, Contestations and Transformations. Cham: Springer International Publishing (2016); Global Justice and Desire. Queering Economy. London [u. a.]: Routledge (2015); mit Maria do Mar Castro Varela: Postkoloniale Theorie. Eine kritische Einführung, 2. komplett überarbeitete und erweiterte Auflage, Bielefeld: Transcript (2015). 